\title{
Inhibition of tumor growth by recombinant vaccinia virus expressing GA733/CO17-1A/EpCAM/KSA/KS1-4 antigen in mice
}

\author{
Jan Zaloudik, ${ }^{1}$ Weiping Li, ${ }^{1}$ Lutz Jacob, ${ }^{1}$ Marie Paule Kieny, ${ }^{2}$ \\ Rajasekharan Somasundaram, ${ }_{1}^{1}$ Bruce Acres, ${ }^{2}$ Hong Song, ${ }^{1}$ Tianqian Zhang, ${ }^{1}$ \\ Jian Li, ${ }^{1}$ and Dorothee Herlyn ${ }^{1}$
}

\author{
${ }^{1}$ The Wistar Institute, Philadelphia, Pennsylvania 19104, USA; and ${ }^{2}$ Transgene S.A., Strasbourg, France.
}

\begin{abstract}
The human colorectal carcinoma (CRC)-associated GA733 antigen (Ag), also named CO17 - 1A/EpCAM/KSA/KS1 - 4, has been a useful target in passive immunotherapy of CRC patients with monoclonal antibody $(\mathrm{mAb})$ and in active immunotherapy with antiidiotypic antibodies or with recombinant protein. These approaches have targeted single epitopes (monoclonal anti-GA733 antibodies and anti-idiotypic antibodies) or extracellular domain epitopes (recombinant protein), primarily by B cells. To determine whether a reagent that induces immunity to a larger number of both B - and T-cell epitopes might represent a superior vaccine, we analyzed the capacity of full - length GA733 Ag expressing multiple potentially immunogenic epitopes and encoded by recombinant vaccinia virus (VV GA733 - 2) to induce humoral, cellular, and/or protective immunity in mice. VV GA733-2 induced Ag-specific antibodies that reacted predominantly to unknown epitopes on the Ag and lysed Ag-positive CRC targets in conjunction with murine peritoneal macrophages as effector cells. Immunized mice developed Ag-specific, proliferative and delayed-type hypersensitive lymphocytes. VV GA733-2 inhibited growth of ras-transformed syngeneic tumor cells expressing the human GA733 Ag in mice. These results suggest the potential of VV GA733-2 as a candidate vaccine for patients with CRC, possibly in combination with recombinant GA733-2-expressing adenovirus, which has been shown to induce cytolytic antibodies and T cells as well as tumor protective effects in mice. The combined vaccine approach may be superior to the use of either vaccine alone in patients who are pre-immune to both viruses.

Cancer Gene Therapy (2002) 9, 382-389 DOI: 10.1038/sj/cgt/7700452
\end{abstract}

Keywords: vaccinia virus; EpCAM; vaccine; tumor growth inhibition

$\mathrm{T}$ he 40-kDa glycoprotein GA733/CO17-1A/EpCAM/ $\mathrm{KSA} / \mathrm{KS} 1-4$ was originally defined by monoclonal antibodies (mAbs) GA733 and CO17-1A, which bind to different epitopes on this antigen $(\mathrm{Ag}) .{ }^{1-3}$ The Ag, referred to hereafter as GA733 Ag, is primarily expressed on colorectal carcinomas (CRCs), gastric, and pancreatic carcinomas. Approximately $85 \%$ of the metastatic CRC lesions from various patients, and $>80 \%$ of the cells within a lesion, are positive. ${ }^{4-7}$ Thus, GA733 is a suitable target for active immunotherapy of these cancers. Although the Ag is also expressed on some normal tissues, such as gastrointestinal, lung, breast, and thyroid tissues, ${ }^{4-7}$ the density of the $\mathrm{Ag}$ is higher on colonic tumor tissues than on normal colon tissues as determined in vitro, ${ }^{1}$ ex vivo, ${ }^{8,9}$ and in vivo. ${ }^{10}$ GA733 is not shed into the patient's blood, which may facilitate tumor targeting by $\mathrm{Ab}$ and/or T cells. ${ }^{11}$ Passive immunotherapy of cancer patients with mAb CO17-1A in doses up to $10 \mathrm{~g}$ has been well tolerated. ${ }^{12} \mathrm{~A}$ phase II trial with 189 patients randomized to treatment with $\mathrm{mAb}$ CO17-

Address correspondence and reprint requests to: Dr Dorothee Herlyn, The Wistar Institute, 3601 Spruce Street, Philadelphia, PA 19104, USA. E-mail:dherlyn@wistar.upenn.edu
1A or placebo control and a median follow - up of 7 years has demonstrated a significant $(P<.01)$ increase in survival of the mAb-treated patients. ${ }^{13}$

In approaches to active immunotherapy targeting the GA733 Ag, patients with cancer mounted humoral ${ }^{14-18}$ and cellular (delayed-type hypersensitive and helper $\mathrm{T}$ cells ${ }^{17,18}$ ) immune responses to the $\mathrm{Ag}$ following the administration of alum-precipitated anti-idiotypic antibodies (Ab2) that functionally mimic the CO17-1A or GA733 epitope. These immune responses may underlie the clinical responses observed in some of the treated patients. ${ }^{14,18}$

The GA733 Ag expresses multiple potentially immunogenic epitopes and in animals, has elicited more effective antitumor immunity than Ab2 mimicking only one epitope on the Ag. ${ }^{19}$ Patients with gastrointestinal carcinoma immunized with alum-precipitated GA733-2E (extracellular domain) protein derived from recombinant baculovirus ${ }^{20}$ showed enhanced humoral and cellular immune responses compared with $\mathrm{Ab} 2$-treated patients. ${ }^{21}$ These clinical trial data clearly demonstrate that induction of active specific immunity to the Ag is possible.

Here, we tested the ability of a recombinant vaccinia virus (VV) expressing full-length GA733 $\mathrm{Ag}^{22}$ (VV GA733-2) to induce humoral, cellular, and protective immunity in mice. 
Unlike GA733-2E, which expresses only extracellular domain epitopes, VV GA733-2 expresses both extracellular and intracellular domain epitopes of the Ag, thus increasing the number of potentially immunogenic epitopes. Importantly, intracellular replication of VV leads to endogenous $\mathrm{Ag}$ expression, which favors CTL induction. Moreover, the GA733 gene product is amplified on replication of the viral vector, thus increasing the Ag load. Whether viral replication also induces cytopathic effects that diminish the yield of $\mathrm{Ag}$ presented remains unknown. However, virus-lysed host cells will likely attract immune and Ag-presenting cells due to local inflammation, necrosis, and apoptosis associated with the self limiting viral infection.

We find that VV GA733-2 elicits tumor cytotoxic antibodies and proliferative and delayed-type hypersensitive lymphocytes. VV GA733-2 also prevents growth of syngeneic Ag-positive tumor cells in mice. The high amino acid sequence homology between the mouse and human GA733 Ag in normal tissues, ${ }^{23}$ and the similarity in normal tissue distribution ${ }^{24}$ makes the mouse a relevant model for experimental active immunotherapy against the GA733 Ag.

\section{Materials and methods}

\section{Tumor cells}

Balb/c 3T3 cells (a gift of Pierre Chambon, Strasbourg, France) were transfected with the ras oncogene in plasmid pVEJB $^{25}$ using $\mathrm{CaPO}_{4}$ precipitation. Cells with the transformed phenotype (loss of contact inhibition) were isolated and grown. Clones were tested for tumorigenicity in Balb/c mice. A tumorigenic clone was transfected with the human GA733-2 cDNA ${ }^{22}$ and selected with G418 (Gibco BRL, Grand Island, NY). FACS analysis revealed $\mathrm{H}-2 \mathrm{k}^{\mathrm{d}}$ expression in $\sim 98 \%$ of the transfectants and GA733 Ag expression in $\sim 86 \%$ of the cells in vitro (not shown). GA733 Ag expression in transfectants injected in vivo followed by ex vivo culture was comparable to $\mathrm{Ag}$ expression by the cells cultured in vitro only (not shown), emphasizing the in vivo stability of the transfected Ag. CT26-GA733-2 and CT-26-ASEN (control) transfectants have been described. ${ }^{26}$ Murine P815 mastocytoma cells, murine Yak natural killer target cells, and human SW1116 CRC cells were obtained from American Type Culture Collection (ATCC, Rockville, MD). Melanoma cell line WM9 has been described. ${ }^{27}$ Murine cell lines were grown in Dulbecco's modified minimal essential medium supplemented with $10 \%$ fetal bovine serum (FBS), whereas human cell lines were grown in Leibovitz L-15 medium supplemented with $10 \%$ FBS.

\section{Antibodies and Ags}

mAbs CO17-1A, GA733, and M77 bind to different epitopes on the GA733 Ag. ${ }^{1-4}$ Anti-CRC mAb CO-029, which binds to an unrelated $\mathrm{CRC} \mathrm{Ag}$, has been described. ${ }^{28}$ Native and recombinant GA733-2E proteins, the latter derived from recombinant baculovirus, were used in in vitro lymphocyte stimulation and enzyme-linked immunosorbent assay (ELISA), respectively, and have been described. ${ }^{20}$
Recombinant $V V$ and adenovirus ( $A d$ ) expressing GA733 Ag

The GA733-2 cDNA was subcloned as an SmaI-PstI fragment into a plasmid under the control of the VV promoter pH5R. VV GA733-2 was generated by homologous recombination of this plasmid in chicken embryo fibroblasts with the genome of VV Copenhagen. ${ }^{29}$ As control VV, VV Copenhagen strain $^{29}$ was used. Ad GA733 2 has been described. ${ }^{30}$

\section{Immunization and tumor challenge}

Six - to 8-week-old female Balb/c mice were used in all immunizations. For antibody induction, seven mice per group were immunized by tail scarification with $10^{6}$ to $10^{8}$ plaque - forming units (PFU) of VV GA733-2 or VV on days 1,8 , and 38. Sera were obtained on days 28 and 60. For induction of proliferative lymphocyte responses, five mice per group were immunized with $10^{7}$ PFU per mouse of VV GA733-2 or VV on days 1,8 , and 38. Two weeks after the last immunization, mice were sacrificed and regional lymph nodes were removed for lymphocyte proliferation assays. For induction of cytolytic T-lymphocyte responses, mice were immunized once or three times (on days 1, 30, and 60) with $10^{7}$ PFU of VV GA733-2 or VV and regional lymph node lymphocytes were collected 2 weeks later. For induction of delayed-type hypersensitivity (DTH) responses, mice (six per group) were immunized with $10^{7}$ PFU of VV GA733-2 or VV once and DTH reaction was determined after 7 days.

Because previous tumor protection studies using murine colon carcinoma CT-26 cells expressing the GA733 Ag revealed significant growth inhibition by low doses of control VV (results not shown), Balb/c 3T3-ras-GA733-2 transfectants were used in the tumor growth inhibition studies. The minimum number of GA733-2-ras transfectants required to induce s.c. tumors in $100 \%$ of the animals was approximately $1 \times 10^{6}$ cells per mouse (not shown). To prevent tumor induction, mice (10 per group) were immunized with $10^{7}$ PFU of VV GA733-2 or VV on days 1,8 , and 38 and injected s.c. with $10^{6} 3 \mathrm{~T} 3$-rasGA733-2 tumor cells on day 52. To inhibit growth of established (vascularized) tumors, mice (eight per group) were injected s.c. with $10^{6}$ tumor cells on day 0 and immunized with VV GA733-2 or VV on days 5, 12, and 43. In both tumor growth inhibition experiments, tumor volumes in cubic millimeters (maximal width $\times$ length $\times$ height) were measured every 3 days with a caliper.

\section{Ab binding assays}

Binding of serum antibodies of immunized mice to tumor cells was determined in mixed hemadsorption assay (MHA) using sheep red blood cells (SRBCs) sensitized with mouse anti-SRBC antibody to which goat antimouse IgG had been bound. ${ }^{20}$ Results were expressed as percent tumor cells binding SRBC. Binding of serum antibodies to GA733-2E protein was tested in ELISA using peroxidase-labeled goat anti-mouse IgG (ICN Biomedicals, Costa Mesa, CA) and substrate (Kirkegaard and Perry Laboratories, Gaithersburg, MD) to detect antibody binding. 


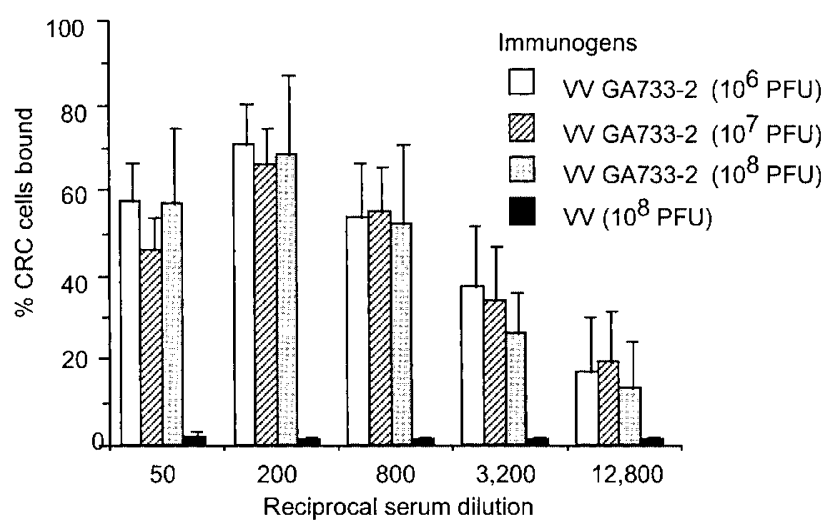

Figure 1 VV GA733-2 elicits Ag-specific cell-binding antibodies in mice. Seven mice per group were immunized by tail scarification with $10^{6}$ to $10^{8}$ PFU of VV GA733-2 or $10^{8}$ PFU VV on days 1,8 , and 38. Sera were obtained on day 60 . Binding of sera to GA733 Ag-positive SW1116 cells was tested in MHA. Sera from VV GA733-2 immunized mice showed significant $(P<.05)$ binding to SW1116 cells compared with control mice immunized with VV at all serum dilutions tested. Data are given as mean \pm SD of seven mice per group.

\section{Ab binding inhibition assays}

Inhibition of mAb 17-1A, GA733, M77, or CO29-10 binding to SW1116 CRC cells by the sera of VV GA733 2- or VV-immunized mice was determined in inhibition radioimmunoassay (RIA). ${ }^{125} \mathrm{I}$-labeled $\mathrm{mAb}$ at concentrations showing $\sim 50 \%$ maximal binding $(10,000 \mathrm{cpm}$ of ${ }^{125} \mathrm{I}-17-1 \mathrm{~A} ; 2,500 \mathrm{cpm}$ of ${ }^{125} \mathrm{I}-\mathrm{GA} 733$, M77 or CO29-10 per well of microtiter plate) was added to SW1116 cells preincubated with various dilutions of sera from immunized mice for 1 hour at room temperature. Binding inhibition of ${ }^{125}$ I-labeled mAbs by either pre-immune or post-immune sera was determined relative to buffer controls.

\section{Antibody-dependent cell-mediated cytotoxicity (ADCC) assays}

Sera from immunized mice were analyzed for ADCC reactivity as described. ${ }^{31}$

\section{Lymphocyte stimulation and proliferation assay}

Lymphocyte proliferation assays were performed as described. ${ }^{26}$ Regional lymph nodes from immunized mice were removed and cell suspensions were prepared. Cells were stimulated at $1 \times 10^{5} /$ well $/ 0.2 \mathrm{~mL}$ of RPMI- 1640 medium (Gibco BRL; supplemented with 10\% FBS, $50 \mu \mathrm{g} / \mathrm{mL}$ gentamycin, $2 \times 10^{-5} \mathrm{M} 2$-mercaptoethanol, $10 \mathrm{mM}$ HEPES, and $2 \mathrm{mM}$-glutamine) with different concentrations $(20$ and $5 \mu \mathrm{g} / \mathrm{mL}$ ) of native full-length GA733 $\mathrm{Ag}$ or with $10^{5} \mathrm{UV}$-irradiated splenocytes infected with $10^{7}$ PFU of VV GA733 - 2 or VV for 3 days. Full-length, rather than extracellular domain, GA733 Ag was used for these studies to detect potential activation of $\mathrm{T}$ lymphocytes by intracellular epitopes present in the VV GA733-2 vaccine. Triplicate cultures were then pulsed overnight with $1 \mu \mathrm{Ci}$ / well of $\left[{ }^{3} \mathrm{H}\right] \mathrm{TdR}$ and harvested by a Harvester 96 system
(Tomec, Orange, CT). Radioactivity bound to the cells was determined in a Matrix $96^{(i \times}$ direct beta counter (Packard, Meriden, CT). Results are expressed as stimulation index (SI).

$$
\mathrm{SI}=\frac{\mathrm{cpm}(\text { experimental cultures })}{\mathrm{cpm}(\text { control cultures })}
$$

\section{Lymphocyte cytotoxicity assay}

For CTL induction, lymphocytes were stimulated with P815 cells infected with $100 \mathrm{PFU} /$ cell of either VV GA733-2 or Ad GA733-2 and irradiated with 10,000 rad (cesium source). Infected P815 cells expressed the GA733 Ag, whereas infected CT26 and 3T3-ras cells did not as determined by FACS analysis (not shown). Target cells included uninfected P815 cells, P815 cells infected with VV GA733-2, Ad GA733-2 or the corresponding control viruses; CT26-GA733-2 or CT-26-ASEN cells; or Yak cells. Balb/c 3T3-ras-GA733-2 cells could not be used as targets because of high spontaneous ${ }^{51} \mathrm{Cr}$ release (see below) of the labeled cells.

Lysis of target cells by mouse lymphocytes was determined in vitro using a standard ${ }^{51} \mathrm{Cr}$-release assay.

\section{DTH assay}

To determine DTH responses, immunized mice were challenged intradermally (i.d.) with $1 \mu \mathrm{g}$ of native GA733 protein into the left ear and with $1 \mu \mathrm{g}$ of bovine serum albumin (BSA) into the right ear. Increase in ear thickness was measured at 18,24 , and 48 hours after challenge.

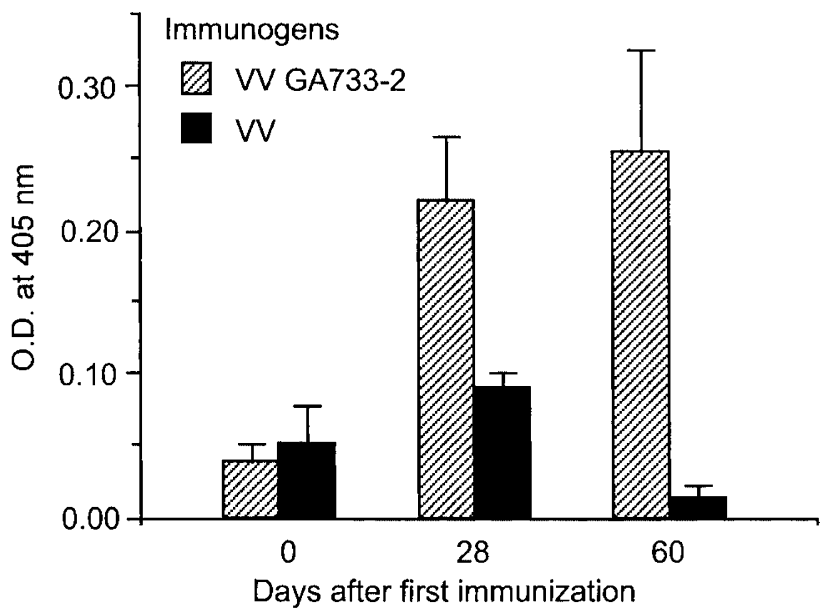

Figure 2 VV GA733-2 elicits Ag-specific antibodies binding specifically to recombinant GA733-2E protein in mice. Mice ( 7 per group) were immunized with $10^{6} \mathrm{PFU}$ of $\mathrm{VV}$ GA733-2 or VV as described in the Figure 1 legend and sera were collected at the indicated days. Binding of sera (diluted 1:50) to GA733-2E extracellular domain protein was determined in ELISA. Sera from experimental mice showed significantly $(P<.05)$ higher binding to GA733-2E compared with sera from control mice on days 28 and 60. The experimental sera did not bind to BSA (not shown). Data are given as mean $\pm S D$ of seven mice per group. 


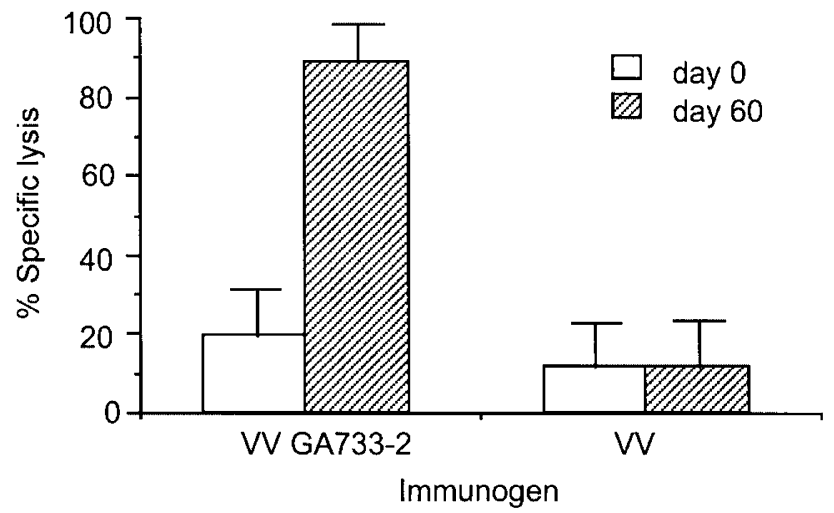

Figure 3 VV GA733-2 elicits Ag-specific ADCC-reactive antibodies in mice. Mice (seven per group) were immunized with $10^{6} \mathrm{PFU}$ of $\mathrm{VV}$ GA733-2 or VV as described in Figure 1 legend. ADCC reactivity of sera (at optimal dilution 1:200) against GA733 Ag-positive SW1116 $\mathrm{CRC}$ cells was tested in a 3 -day $\left[{ }^{3} \mathrm{H}\right]$ thymidine-incorporation assay using thioglycolate-elicited murine peritoneal macrophages as effector cells. Specific lysis by sera of VV GA733-2-immunized mice on day 60 post-immunization was significantly $(P<.05)$ higher than the corresponding pre-immunization value and the value obtained in VV-immunized mice on day 60 . Sera did not significantly lyse Ag-negative WM- 9 melanoma cells ( not shown). Data are given as mean $\pm \mathrm{SD}$ of seven mice per group.

\section{Statistical analyses}

Experimental and control values were compared using the Student's $t$ test. Differences between experimental and control values were considered significant at $P<.05$.

\section{Results}

Antibody responses in VV GA733-2-immunized mice

Sera of mice immunized three times with VV GA733-2 showed significant binding $(P<.05)$ to GA733 Ag-positive

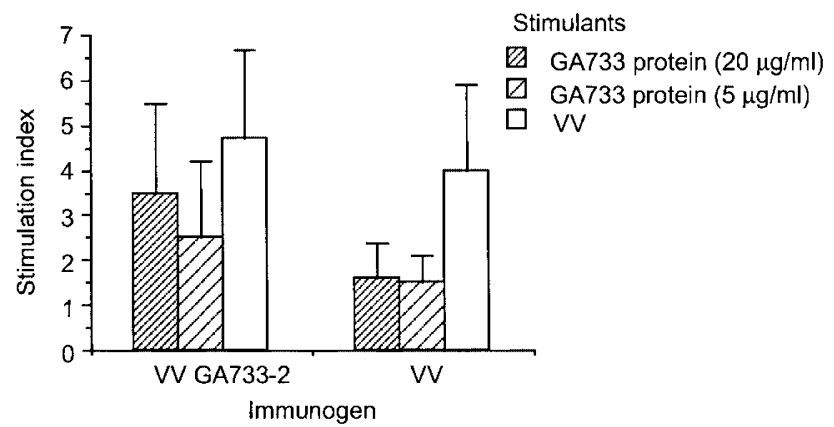

Figure 4 VV GA733-2 elicits Ag-specific lymphoproliferative responses in mice. Mice (five per group) were immunized with $10^{7}$ PFU per mouse of VV GA733-2 or VV on days 1, 8, and 38. Two weeks after the last immunization, mice were sacrificed and regional lymph nodes were removed and stimulated with native GA733 protein for 3 days. Lymphocyte proliferation was tested in $\left[{ }^{3} \mathrm{H}\right]$ thymidineincorporation assay. Lymphoproliferative responses of mice immunized with VV GA733-2 and stimulated with $20 \mu \mathrm{g} / \mathrm{mL}$ native GA733 protein were significantly $(P<.05)$ higher than those of control VVimmunized mice stimulated with the same amount of protein. Lymphocytes from both experimental and control mice proliferated in response to VV (positive control) stimulation. Data are given as mean $\pm S D$ of five mice per group.
SW1116 cells in MHA compared with sera of VV-immunized control mice (Fig 1). SW1116 cells, which naturally express GA733 Ag at a density of $\sim 10^{6}$ molecules per cell, ${ }^{2,32}$ were used as targets instead of 3T3-ras-GA733 transfectants on which $\mathrm{Ag}$ density is higher $\left(>10^{6}\right.$ molecules/cell), potentially biasing interpretation of the results. There was no virus dose dependency $\left(10^{6}, 10^{7}, 10^{8} \mathrm{PFU}\right)$ of the immune response. Antibody titers were $>1: 12,800$. These antibodies did not bind to Ag-negative WM-9 melanoma cells in MHA or to the CO17-1A, GA733 or M77 epitopes in inhibition RIA (not shown). ELISA analysis revealed significant $(P<.05$ vs. control sera $)$ binding of the elicited antibodies to GA733-2E protein (Fig 2), but not to BSA (not shown). Serum antibodies elicited by VV GA733-2 mediated significant $(P<.05)$ lysis of GA733 Ag-positive SW1116 cells compared with control sera (Fig 3 ) or to WM9 target cells (not shown).

Presence of proliferative and absence of cytolytic lymphocyte responses in VV GA733-2 immunized mice

Mice were immunized three times with VV GA733-2 or VV and regional lymph node lymphocytes were stimulated with native GA733 protein or with splenocytes infected with VV GA733-2 or VV, followed by UV irradiation. Comparison of the proliferative activities of lymphocytes from VV GA733 2 and control immunized mice revealed Ag-specific significant $(P<.05)$ proliferation only when native GA733 protein was used as stimulant (Fig 4). Stimulation of lymphocytes with VV-infected splenocytes (positive control) induced proliferative lymphocyte responses in experimental and control mice, which were of similar magnitude

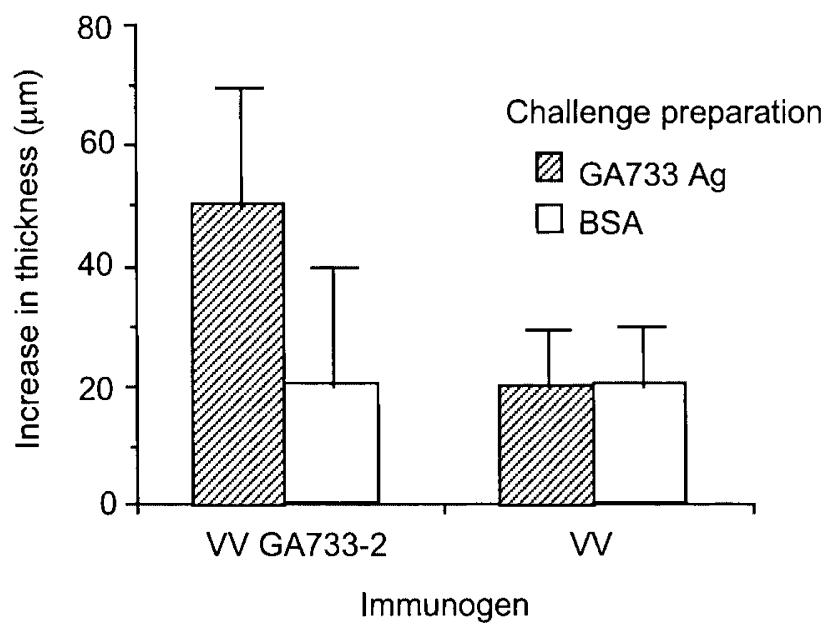

Figure 5 VV GA733-2 elicits Ag-specific DTH responses in mice. For induction of DTH responses, mice (six per group) were immunized with $10^{7} \mathrm{PFU}$ once and DTH reaction was determined after 7 days. Mice were challenged i.d. with $1 \mu \mathrm{g}$ of native GA733 protein into the left ear and with $1 \mu \mathrm{g}$ of BSA into the right ear. Increase in ear thickness was measured at 18,24 , and 48 hours after challenge; values are shown for the 18 hour time point. DTH reactions of VV GA733-2 immunized mice to challenge with native GA733 Ag were significantly $(P<.05)$ higher than the responses to BSA challenge or the responses of VV immunized mice challenged with GA733 Ag. Data are given as mean \pm SD of six mice per group. 


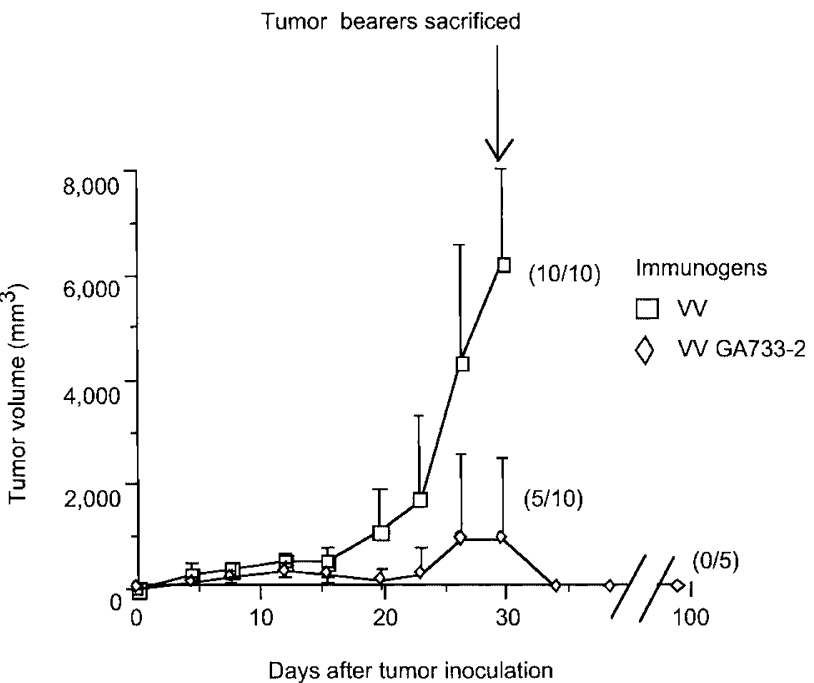

Figure 6 VV GA733-2 inhibits growth of Balb/c 3T3-ras-GA733-2 tumor cells in mice. Mice (10 per group) were immunized with $10^{7}$ PFU of VV GA733-2 or VV on days 1, 8, and 38, and injected s.c. with $10^{6}$ 3T3-ras-GA733-2 tumor cells on day 52. Tumor volumes (maximal width $\times$ length $\times$ height) were measured with a caliper. Control mice and 5 of 10 experimental mice were sacrificed on day 30 because of large tumor burden. Tumor growth was significantly $(P<.05)$ inhibited by VV GA733 compared with VV on day 30 . Five of the 10 experimental mice were tumor free on day 100. Ratios are numbers of animals with tumors to total number. Data are given as mean \pm SD of 10 mice per group.

compared to the Ag-specific proliferative lymphocyte responses observed in experimental mice after lymphocyte stimulation with GA733-2 protein (Fig 4) or VV GA733 2 -infected splenocytes (not shown).

For CTL induction, mice were immunized once or three times, and immune lymphocytes were stimulated with GA733-2E or P815 cells infected with VV GA733-2 or Ad GA733-2. Syngeneic virus-infected splenocytes were not used as stimulators because they did not induce $\mathrm{Ag}$ specific lymphoproliferative immune responses (see above). Lysis of various target cells (uninfected P815 cells or P815 cells infected with VV GA733-2, Ad GA733-2 or the corresponding control viruses; CT26-GA733-2 or CT-26ASEN cells; or Yak cells ) was determined. However, no Agspecific CTL responses were detected in any of the mice immunized with VV GA733-2. In positive control experiments, lymphocytes from VV GA733-2-immunized mice stimulated with VV GA733-2-infected P815 cells significantly lysed VV or VV GA733-2-infected P815 target cells compared with P815 cells. However, lysis of the VV GA733-2-infected target cells was no greater than lysis of VV-infected target cells ( $88 \%$ and $75 \%$ specific lysis of P815 cells infected with VV and VV GA733-2, respectively; $20 \%$ lysis of uninfected P815 cells). Thus, anti-VV CTL, but not GA733 Ag-specific CTL responses were obtained.

\section{DTH responses}

Mice were immunized once with VV GA733-2 or VV and challenged i.d. with native GA733 Ag or BSA. Increase in ear thickness was measured at 18,24 , and 48 hours after challenge. DTH reactions of VV GA733-2-immunized mice to challenge with native GA733 Ag were significantly $(P<.05)$ higher than the responses to BSA challenge or the responses of VV-immunized mice challenged with GA733 Ag (Fig 5).

\section{Inhibition of tumor growth}

In mice immunized three times with VV GA733-2 and challenged with 3T3-ras-GA733-2 cells 12 days after the last immunization, tumor growth was significantly $(P<.05)$ inhibited compared with control mice (Fig 6). The tumors grew exponentially in all 10 control mice, which were sacrificed on day 30 of the experiment because of large tumor burden $(>1 \mathrm{~cm}$ maximal diameter $)$. In contrast, only 5 of 10 experimental mice showed tumor growth on day 30 , and these 5 mice were also sacrificed because of large tumor burden. The 5 surviving mice still showed no tumor growth on day 100 when the experiment was terminated. However, VV GA733-2 was unable to inhibit 5-day established tumors in mice $(P>.05$; not shown $)$.

\section{Discussion}

The GA733-2 Ag expressed in recombinant VV is a promising vaccine reagent in mice. VV GA733-2 induced humoral, cellular, and protective immunity specific for the $\mathrm{Ag}$ in mice, whereas no significant immunity was induced by the control virus. VV GA733-2 induced antibodies that specifically bound to and lysed $\mathrm{Ag}$-positive tumor cells in ADCC. Antibody epitope analysis revealed that none of the three epitopes (CO17-1A, GA733, and M77) tested was recognized by VV GA733-2-immunized mice. Thus, the humoral immune responses to VV GA733-2 included primarily antibodies directed to CO17-1A-, GA733- and M77-unrelated epitopes. In contrast, mice immunized with human CRC cells produced antibodies to the CO17-1A and GA733 epitopes. ${ }^{1,2}$ Therefore, immunologic presentation of the GA733 Ag expressed by human tumor cells differs from $\mathrm{Ag}$ presentation in the $\mathrm{VV}$ vector.

VV GA733-2 induced Ag-specific cellular immune responses consisting of proliferating and DTH-reactive lymphocytes in mice. However, GA733 Ag-specific CTL responses were not detected, although immunized mice produced strong anti-VV CTL responses. This was surprising because after infection of host cells with recombinant viruses, the foreign $\mathrm{Ag}$ is endogenously produced by the cells and usually presented to host immune lymphocytes in the context of major histocompatibility (MHC) class I Ag. ${ }^{33}$ Thus, CTL, which may play an important role in the control of cancer ${ }^{34}$ and which recognize endogenously produced $\mathrm{Ag}$, usually in association with MHC class I molecules, ${ }^{35}$ are likely to be induced by recombinant vaccines employing viral vectors. Indeed, several studies have shown that recombinant VV expressing tumor-associated $\mathrm{Ag}$ can induce Ag-specific CTL responses in experimental animals. ${ }^{36-48}$ The GA733 Ag expresses amino acid sequences that could potentially associate with murine $\mathrm{H}-2 \mathrm{k}^{\mathrm{d}}$ (amino acid residues 173-181, 185-193, and 88-96). Furthermore, Ad GA733-2 induced GA733 Ag-specific CTL responses. ${ }^{30}$ It is possible that following VV GA733-2 
immunizations in mice, the GA733 CTL epitopes were not functionally expressed by $\mathrm{Ag}$-presenting cells or not adequately presented to $\mathrm{T}$ cells.

VV GA733-2 prevented the growth of GA733 Agpositive, syngeneic tumor cells but not growth of established tumors. In contrast, Ad GA733-2 inhibited growth of established tumors in mice, although murine CT-26 colon carcinoma cells were used in that study $;^{30}$ those cells were not used in the present study because their growth was nonspecifically inhibited by control VV. Because $\mathrm{Ad}$ GA733-2, but not VV GA733-2, elicited CTL responses in mice, these responses may be crucial for the inhibition of established tumors.

Our results obtained in vitro with cells from mice immunized according to protocols optimized for induction of humoral and cellular immunity suggest that GA733-2 Ag-specific proliferative and/or DTH-reactive lymphocytes, and/or ADCC-reactive antibodies contribute to tumor growth inhibition in vivo. The DTH-reactive lymphocyte responses most likely are mediated by T-helper type 1 (Th1) cells ${ }^{49,50}$ which may have activated effector cells of the innate immune system, such as monocytes/macrophages, natural killer cells, neutrophils, and/or eosinophils at the tumor site, leading to tumor destruction. In addition, viral infection may have activated the innate immune system.

Recombinant VV expressing various tumor-associated Ags have been shown to control tumor growth in laboratory animals. Tumor growth inhibition was demonstrated with prophylactic immunizations, ${ }^{36,37,39,41-44,46,48,51-60}$ with therapeutic immunizations, ${ }^{38,40,45,61-64}$ or with both prophylactic and therapeutic immunizations. ${ }^{42,44,46,57-59}$ In some of those studies, costimulatory molecules or cytokines were used to enhance vaccine effects. ${ }^{44,45,59,64}$ These studies have suggested a role for antibodies, ${ }^{36-41,43,47,48,51-53,60,63,64}$ CTL, ${ }^{36-48}$ and delayed-type hypersensitive T cells, ${ }^{36,37,46,53}$ although the role of these factors was not directly demonstrated by in vivo cell depletion or adoptive cell transfer. The mouse models targeting human carcinoembryonic Ag (CEA) and MUC- 1 with VV vaccines, ${ }^{36,39-41,43,45-48,52,63}$ similar to the human GA733 model used in the present study, include Ags that are expressed by tumors and by normal tissues. Mice express homologues of these human Ags on their normal tissues ${ }^{23,65,66}$ and thus provide a clinically relevant model. VV CEA has induced CTL and clinical responses in patients with CRC. ${ }^{67-70}$

The VV GA733 - 2 construct described here is a candidate vaccine for patients with CRC. However, a concern pertaining to the use of recombinant $\mathrm{VV}$ vaccines in patients is whether previous exposure to the virus (most patients $>50$ years of age are seropositive for VV) negatively affects induction of Ag-specific immunity by recombinant $\mathrm{VV}^{71}$ although repeated immunization of CRC patients with VV CEA induced immune responses in the majority of patients. $^{71}$

VV GA733-2 may be especially valuable for combined vaccinations of cancer patients with Ad GA733-2, which induces humoral, cellular, and protective immune responses in mice. ${ }^{30}$ Sequential immunization with different recombinant viruses has shown superior antitumor effects compared with immunizations with a single virus, ${ }^{38,39}$ due possibly to the enhanced boosting of the tumor Ag-specific compared with virus-specific immune responses when different viruses are used in combination. The combined vaccine approach is particularly attractive for patients with cancer who are pre-immune to Ad and/or VV. Moreover, VV GA733 - 2 and Ad GA733 - 2 vaccines differ in their capacity to induce CTL, DTH, and protective responses against established tumors in mice, further pointing to the need to evaluate the combined effect of these reagents.

A clinical trial with Ad GA733-2 is in preparation and if promising, will be followed by a trial combining both $\mathrm{Ad}$ GA733 - 2 and VV GA733 -2. We have already demonstrated that active immunotherapy of CRC patients with $\mathrm{Ab} 2$ mimicking the CO17-1A or GA733 epitope or with extracellular domain GA733-2E protein, all precipitated with alum, induced epitope-specific humoral, cellular, and, possibly, protective immunity. ${ }^{15-18,21}$ However, singleepitope Ab2 vaccines and extracellular domain GA733-2E protein are expected to induce less potent antitumor immunity compared to full-length $\mathrm{Ag}$ vaccines, which express multiple, potentially immunogenic epitopes. Thus, Ab2 mimicking the GA733 epitope ${ }^{26}$ and extracellular domain protein GA733-2 $\mathrm{E}^{19}$ did not protect mice against challenge with CT26-GA733-2 tumor cells, in marked contrast to the significant antitumor effects of VV GA733 -2 described here and Ad GA733-2 described previously, ${ }^{30}$ which both express full-length GA733 Ag.

\section{Acknowledgments}

This work was supported in part by Grants CA 10815, CA 60595, CA 74294, and CA 29797 from the National Institutes of Health, and a grant from Ajinomoto, Japan. We thank Dr Eva Gönczöl for critically reviewing this manuscript.

\section{References}

1. Herlyn M, Steplewski Z, Herlyn D, et al. Colorectal carcinomaspecific antigen: detection by means of monoclonal antibodies. Proc Natl Acad Sci USA. 1979;76:1438-1442.

2. Herlyn D, Herlyn M, Ross AH, et al. Efficient selection of human tumor growth-inhibiting monoclonal antibodies. J Immunol Methods. 1984;73:157-167.

3. Ross AH, Herlyn D, Iliopoulos D, et al. Isolation and characterization of a carcinoma-associated antigen. Biochem Biophys Res Commun. 1986;135:297-303.

4. Göttlinger HG, Funke I, Johnson JP, et al. The epithelial cell surface antigen 17-1A, a target for antibody-mediated tumor therapy: its biochemical nature, tissue distribution and recognition by different monoclonal antibodies. Int $J$ Cancer. 1986;38:47-53

5. Herlyn M, Steplewski Z, Herlyn D, et al. CO17-1A and related monoclonal antibodies: their production and characterization. Hybridoma. 1986;5(Suppl):S3-S10.

6. Shetye J, Frödin J-E, Christensson B, et al. Immunohistochemical monitoring of metastatic colorectal carcinoma in patients treated with monoclonal antibodies (MAb 17-1A). Cancer Immunol Immunother. 1988;27:154-162. 
7. Shetye J, Christensson B, Rubio C, et al. The tumor-associated antigens BR55-2, GA73-3, and GICA 19-9 in normal and corresponding neoplastic human tissues, especially gastrointestinal tissues. Anti-Cancer Res. 1989;9:395-404.

8. Sears HF, Herlyn D, Herlyn M, et al. Ex vivo perfusion of a tumor-containing colon with monoclonal antibody. J Surg Res. 1981;31:145-150.

9. Sears HF, Herlyn D, Herlyn M, et al. Ex vivo perfusion of human colon with monoclonal anti-colorectal cancer antibodies. Cancer. 1982;49:1231-1235.

10. Mach J-P, Chatal J-F, Lumbroso J-D, et al. Tumor localization in patients by radiolabeled monoclonal antibodies against colon carcinoma. Cancer Res. 1983;43:5593-5600.

11. Powe J, Herlyn D, Alavi A, et al. Radioimmunodetection of human tumor xenografts by monoclonal antibodies correlates with antibody density and affinity. In: Button K, Donato L, eds. Immunoscintigraphy. New York: Gordon \& Breach Science Publishers; 1985:135-152.

12. LoBuglio AF, Saleh MN, Lee J, et al. Phase I trial of multiple large doses of murine monoclonal antibody CO17-1A. I. Clinical aspects. J Natl Cancer Inst. 1988;80:932-936.

13. Riethmüller G, Holtz E, Schlimok G, et al. Monoclonal antibody therapy for resected Dukes' C colorectal cancer: seven-year outcome of a multicenter randomized trial. J Clin Oncol. 1998;16:1788-1794.

14. Herlyn D, Wettendorff M, Schmoll E, et al. Anti-idiotype immunization of cancer patients: modulation of the immune response. Proc Natl Acad Sci USA. 1987;84:8055-8059.

15. Loibner H, Plot R, Rot A, et al. Immunoreactivity of patient with colorectal cancer metastasis after immunization with antiidiotypes. Lancet. 1990;335:171.

16. Herlyn D, Benden A, Kane M, et al. Anti-idiotype cancer vaccines: preclinical and clinical studies. In Vivo. 1991;5:615624.

17. Herlyn D, Harris D, Zaloudik J, et al. Immunomodulatory activity of monoclonal anti-idiotypic antibody to anti-colorectal carcinoma antibody $\mathrm{CO} 17-1 \mathrm{~A}$ in animals and patients. J Immunother. 1994;15:303-311.

18. Somasundaram R, Zaloudik J, Jacob L, et al. Induction of Tand B-cell immunity in colon carcinoma patients by antiidiotypic antibody. J Immunol. 1995;155:3253-3261.

19. Maruyama H, Zaloudik J, Li W, et al. Point and counterpoint. Cancer vaccines: single epitope anti-idiotype vaccine versus multiple epitope antigen vaccine. Cancer Immunol Immunother. 2000;49:123-132.

20. Strassburg CP, Kasai Y, Seng BA, et al. Baculovirus recombinant expressing a secreted form of a transmembrane carcinoma-associated antigen. Cancer Res. 1992; $52: 815-821$.

21. Staib L, Birebent B, Somasundaram R, et al. Immunogenicity of recombinant GA733-2E antigen (CO17-1A, EGP, KS1 -4, KSA, Ep-CAM) in gastrointestinal carcinoma patients. Cancer Gene Ther. 2002;9:142-148.

22. Szala S, Froehlich M, Scollon M, et al. Molecular cloning of cDNA for the carcinoma-associated antigen GA733-2. Proc Natl Acad Sci USA. 1990;87:3542-3546.

23. Bergsagel PL, Victor-Kobrin C, Timblin CR, et al. A murine cDNA encodes a pan-epithelial glycoprotein that is also expressed on plasma cells. J Immunol. 1992;148:590-596.

24. Zaloudik J, Basak S, Nesbit M, et al. Expression of an antigen homologous to the human CO17-1A/GA733 colon cancer antigen in animal tissues. Br J Cancer. 1997;76:909-916.

25. Land H, Parada LF, Weinberg RA. Tumorigenic conversion of primary embryo fibroblasts requires at least two cooperating oncogenes. Nature. 1983;304:596-602.
26. Maruyama H, Benden A, Li W, et al. Monoclonal anti-idiotypic antibody functionally mimics the human gastrointestinal carcinoma epitope GA733. Int J Cancer. 1996;65:547-553.

27. Herlyn M, Balaban G, Bennicelli J, et al. Primary melanoma cells of the vertical growth phase: similarities to metastatic cells. J Natl Cancer Inst. 1985;74:283-289.

28. Koprowski H, Steplewski Z, Mitchell K, et al. Colorectal carcinoma antigens detected by hybridoma antibodies. Somatic Cell Genet. 1979;5:957-972.

29. Kieny MP, Lathe R, Drillien R, et al. Expression of rabies virus glycoprotein from a recombinant vaccinia virus. Nature. 1984;312:163-166.

30. Li W, Berencsi K, Basak S, et al. Human colorectal cancer (CRC) antigen CO17-1A/GA733 encoded by recombinant adenovirus inhibits growth of established CRC cells in mice. $J$ Immunol. 1997;159:763-769.

31. Herlyn D, Koprowski H. IgG2a monoclonal antibodies inhibit human tumor growth through interaction with effector cells. Proc Natl Acad Sci USA. 1982;79:4761-4765.

32. Powe J, Herlyn D, Alavi A, et al. Radioimmunodetection of human tumor xenografts by monoclonal antibodies correlates with antibody density and affinity. In: Button K, Donato L, eds. Immunoscintigraphy. New York: Gordon \& Breach Science Publishers; 1985:135-152.

33. Zhai Y, Yang JC, Kawakami Y, et al. Antigen-specific tumor vaccines. J Immunol. 1996;156:700-710.

34. Houghton AN. On course for a cancer vaccine. Lancet. 1995;345:1384-1385.

35. Townsend ARM, Rothbard J, Gotch FM, et al. The epitopes of influenza nucleoprotein recognized by cytotoxic $\mathrm{T}$ lymphocytes can be defined with short synthetic peptides. Cell. 1986;44:959-968.

36. Kantor J, Irvine K, Abrams S, et al. Antitumor activity and immune responses induced by a recombinant carcinoembryonic antigen-vaccinia virus vaccine. J Natl Cancer Inst. 1992;84:1084-1091.

37. Estin CD, Stevenson US, Plowman GD, et al. Recombinant vaccinia virus vaccine against the human melanoma antigen p97 for use in immunotherapy. Proc Natl Acad Sci USA. 1988;85:1052-1056.

38. Irvine KR, Chamberlain RS, Shulman EP, et al. Enhancing efficacy of recombinant anticancer vaccines with prime/boost regimens that use two different vectors. $J$ Natl Cancer Inst. 1997;89:1595-1601.

39. Hodge JW, McLaughlin JP, Kantor JA, et al. Diversified prime and boost protocols using recombinant vaccinia virus and recombinant non-replicating avian pox virus to enhance T-cell immunity and antitumor responses. Vaccine. 1997;15: 759-768.

40. Grosenbach DW, Barrientos JC, Schlom J, et al. Synergy of vaccine strategies to amplify antigen-specific immune responses and antitumor effects. Cancer Res. 2001;61:44974505.

41. Bei R, Kantor J, Kashmiri SVS, et al. Enhanced immune responses and anti-tumor activity by baculovirus recombinant carcinoembryonic antigen (CEA) in mice primed with the recombinant vaccinia CEA. J Immunother. 1994;16:275-282.

42. Wang M, Bronte V, Chen PW, et al. Active immunotherapy of cancer with a nonreplicating recombinant fowlpox virus encoding a model tumor-associated antigen. J Immunol. 1995; $154: 4685-4692$.

43. Acres RB, Hareuveni M, Balloul J-M, et al. Vaccinia virus MUC1 immunization of mice: immune response and protection against the growth of murine tumors bearing the MUC1 antigen. J Immunother. 1993;14:136-143. 
44. Bronte V, Tsung K, Rao JB, et al. IL-2 enhances the function of recombinant poxvirus - based vaccines in the treatment of established pulmonary metastases. J Immunol. 1995;154: 5282-5292.

45. McLaughlin JP, Schlom J, Kantor JA, et al. Improved immunotherapy of a recombinant carcinoembryonic antigen vaccinia vaccine when given in combination with interleukin2. Cancer Res. 1996;56:2361-2367.

46. Schlom J, Kantor J, Abrams, S, et al. Strategies for the development of recombinant vaccines for the immunotherapy of breast cancer. Breast Cancer Res Treat. 1996;38:27-39.

47. Acres B, Apostolopoulos V, Balloul JM, et al. MUC1-specific immune responses in human MUC1 transgenic mice immunized with various human MUC1 vaccines. Cancer Immunol Immunother. 2000;48:588-594.

48. Kass E, Schlom J, Thompson J, et al. Induction of protective host immunity to carcinoembryonic antigen (CEA), a self-antigen in CEA transgenic mice, by immunizing with a recombinant vaccinia-CEA virus. Cancer Res. 1999;59:676-683.

49. Mosmann TR, Coffman RL. Th1 and Th2 cells: different patterns of lymphokine secretion lead to different functional properties. Annu Rev Immunol. 1989;7:145-173.

50. Suzuki M, Iwashiro M, Takatsuki F, et al. Reconstitution of anti-tumor effects of lentinan in nude mice: roles of delayedtype hypersensitivity reaction triggered by CD4-positive Tcell clone in the infiltration of effector cells into tumor. Jpn $J$ Cancer Res. 1994;85:409-417.

51. Bernards R, Destree A, McKenzie S, et al. Effective tumor immunotherapy directed against an oncogene-encoded product using a vaccinia virus vector. Proc Natl Acad Sci USA. 1987;84:6854-6858.

52. Hareuveni M, Gautier C, Kieny M-P, et al. Vaccination against tumor cells expressing breast cancer epithelial tumor antigen. Proc Natl Acad Sci USA. 1990;87:9498-9502.

53. Meneguzzi G, Kieny MP, Lecocq J-P, et al. Vaccinia recombinants expressing early bovine papilloma virus (PBV1) proteins: retardation of BPV1 tumour development. Vaccine. 1990;8:199-204.

54. Ito T, Wang D-Q, Maru M, et al. Antitumor efficacy of vaccinia virus-modified tumor cell vaccine. Cancer Res. 1990;50:6915-6918.

55. Meneguzzi G, Cerni C, Kieny MP, et al. Immunization against human papillomavirus type 16 tumor cells with recombinant vaccinia viruses expressing E6 and E7. Virology. 1991;81:6269.

56. Irvine KK, Abrams S, Snoy P, et al. Immunogenicity and safety of a recombinant vaccinia virus vaccine expressing the carcinoembryonic antigen gene in a nonhuman primate. Cancer Res. 1992;52:6917-6925.

57. Lathe R, Kieny MP, Gerlinger P, et al. Tumour prevention and rejection with recombinant vaccinia. Nature. 1987;326: $878-880$.

58. Lin K-Y, Guarnieri FG, Staveley-O'Carroll KF, et al. Treatment of established tumors with a novel vaccine that enhances major histocompatibility class II presentation of tumor antigen. Cancer Res. 1996;56:21-26.

59. Chamberlain RS, Carroll MW, Bronte V, et al. Costimulation enhances the active immunotherapy effect of recombinant anticancer vaccines. Cancer Res. 1996;56:2832-2836.

60. Rao JB, Chamberlain RS, Bronte V, et al. IL-12 is an effective adjuvant to recombinant vaccinia virus - based tumor vaccines. J Immunol. 1996;156:3357-3365.

61. Overwijk WW, Lee DS, Surman DR, et al. Vaccination with a recombinant vaccinia virus encoding a "self" antigen induces autoimmune vitiligo and tumor cell destruction in mice: requirement for CD4(+) T lymphocytes. Proc Natl Acad Sci USA. 1999;16:2982-2987.

62. McCabe BJ, Irvine KR, Nishimura MI, et al. Minimal determinant expressed by a recombinant vaccinia virus elicits therapeutic antitumor cytolytic $\mathrm{T}$ lymphocyte responses. Cancer Res. 1995;55:1741-1747.

63. Kaufman H, Schlom J, Kantor J. A recombinant vaccinia virus expressing human carcinoembryonic antigen (CEA). Int $J$ Cancer. 1991;48:900-907.

64. Rosales C, Graham VV, Rosas GA, et al. A recombinant vaccinia virus containing the papilloma $\mathrm{E} 2$ protein promotes tumor regression by stimulating macrophage antibodydependent cytotoxicity. Cancer Immunol Immunother. 2000; 49:347-360.

65. Spicer AP, Parry G, Patton S, et al. Molecular-cloning and analysis of the mouse homolog of the tumor-associated mucin, MUC1, reveals conservation of potential O-glycosylation sites, transmembrane, and cytoplasmic domains and a loss of minisatellite-like polymorphism. J Biol Chem. 1991;266: 15099-15109.

66. Beauchemin N, Turbide C, Afar D, et al. A mouse analogue of the human carcinoembryonic antigen. Cancer Res. 1989;49: 2017-2021.

67. Conry RM, Khazaeli MB, Saleh MN, et al. Phase I trial of a recombinant vaccinia virus encoding carcinoembryonic antigen in metastatic adenocarcinoma: comparison of intradermal versus subcutaneous administration. Clin Cancer Res. 1999;5:2330-2337.

68. Eder JP, Kantoff PW, Roper K, et al. A phase I trial of a recombinant vaccinia virus expressing prostate-specific antigen in advanced prostate cancer. Clin Cancer Res. 2000;6:16321638.

69. Conry RM, Allen KO, Lee S, et al. Human autoantibodies to carcinoembryonic antigen (CEA) induced by a vaccinia-CEA vaccine. Clin Cancer Res. 2000;6:34-41.

70. Tsang K, Zaremba Y, Nieroda S, et al. Generation of human cytotoxic $\mathrm{T}$ cells specific for human carcinoembryonic antigen epitopes from patients immunized with recombinant vacciniaCEA vaccine. J Natl Cancer Inst. 1995;87:949-990.

71. Cooney EL, Collier AC, Greenberg PD, et al. Safety of an immunological response to a recombinant vaccinia virus vaccine expressing HIV envelope glycoprotein. Lancet. 1991;337:567-572. 